\title{
Разработка и исследование туннельных $p-i-n$-диодов GaAs/AIGaAs для многопереходных преобразователей мощного лазерного излучения
}

\author{
(C) В.С. Калиновский, Е.В. Контрош, Г.В. Климко, С.В. Иванов, В.С. Юферев, Б.Я. Бер, \\ Д.Ю. Казанцев, В.М. Андреев
}

Физико-технический институт им. А.Ф. Иоффе Российской академии наук, 194021 Санкт-Петербург, Россия

E-mail: vitak.sopt@mail.ioffe.ru

Поступила в Редакцию 29 октября 2019 г.

В окончательной редакции 5 ноября 2019 г.

Принята к публикации 5 ноября 2019 г.

Создание соединительных туннельных диодов с высокой плотностью пикового туннельного тока, превышающей плотность тока короткого замыкания фотоактивных $p-n$-переходов, является важной задачей при разработке многопереходных фотопреобразователей $\left(\mathrm{A}^{\mathrm{III}} \mathrm{B}^{\mathrm{V}}\right)$ мощного оптического излучения. На основе результатов численного моделирования вольт-амперных характеристик туннельных диодов предложен способ повышения плотности пикового туннельного тока путем включения между вырожденными слоями туннельного диода тонкого нелегированного $i$-слоя толщиной в несколько нанометров. Методом молекулярнопучковой эпитаксии выращены структуры $p-i-n-\mathrm{GaAs} / \mathrm{Al}_{0.2} \mathrm{Ga}_{0.8} \mathrm{As}$ туннельных соединительных диодов со значениями пиковой плотности туннельного тока до $200 \mathrm{~A} / \mathrm{cm}^{2}$.

Ключевые слова: туннельный диод, квантовое туннелирование, вольт-амперная характеристика, многопереходный фотопреобразователь, молекулярно-пучковая эпитаксия.

DOI: 10.21883/FTP.2020.03.49034.9298

\section{1. Введение}

С развитием радиофотоники и концентраторной фотовольтаики растет необходимость в разработке высокоэффективных фотоэлектрических устройств, преобразующих мощное оптическое излучение. К данным устройствам относятся, например, многопереходные фотопреобразователи мощного лазерного излучения и концентраторные многопереходные солнечные элементы на основе полупроводниковых соединений $\mathrm{A}^{\mathrm{III}} \mathrm{B}^{\mathrm{V}}$. Рекордное значение коэффициента полезного действия (кпд) многопереходных солнечных элементов в настоящее время составляет $>46 \%$ (при концентрации $\geq 500$, AM1.5) [1,2], в то время как кпд однопереходных фотопреобразователей лазерного излучения превышает $50 \%[3,4]$. Коэффициент полезного действия многопереходных фотопреобразователей существенно зависит от параметров соединительных туннельных диодов (ТД). Для обеспечения максимального кпд соединительные туннельные диоды должны обладать высокой оптической прозрачностью, плотностью пикового туннельного тока, превышающей плотность тока короткого замыкания фотопреобразователя, и низким дифференциальным сопротивлением для минимизации падения напряжения на ТД. Высокая оптическая прозрачность ТД достигается путем снижения толщин вырожденных слоев ТД до 10 нм и использования широкозонных полупроводниковых материалов. Однако при столь малых толщинах происходит практически полное обеднение вырожденных $n$-слоев. В результате положение дна зоны проводимости начинает определяться прилегающим менее легированным слоем, что приводит к уменьшению диапазона энергий, при которых возможно туннелирование электронов при прямом смещении на диоде. Использование же более широкозонных материалов способствует снижению вероятности квантового туннелирования [5]. Все эти факторы отрицательно сказываются на величине плотности пикового тока ТД.

С другой стороны, увеличение плотности пикового туннельного тока в соединительных ТД на основе $\mathrm{Ga}(\mathrm{Al}) \mathrm{As}$ в многопереходных структурах ограничено сложностью реализации $n$-типа проводимости. Достижение концентрации электронов, превышающей $2 \cdot 10^{19} \mathrm{~cm}^{-3}$, с использованием любого метода введения легирующей примеси $(\mathrm{Si}, \mathrm{Te})$ является сложной технологической задачей [6-8]. При создании $p$-типа проводимости в GaAs на основе элементов второй группы (Be, $\mathrm{Mg}, \mathrm{Zn})$ с помощью технологии молекулярно-пучковой эпитаксии (МПЭ) удается достигать концентрации дырок $1 \cdot 10^{20} \mathrm{~cm}^{-3}[9]$. Однако, согласно $[10,11]$, подъем концентрации, например, акцепторной примеси Ве до $1 \cdot 10^{18} \mathrm{~cm}^{-3}$ и выше влечет за собой аномальную диффузию атомов Ве в сторону границы $p^{++}-n^{++}$-перехода. Это перераспределение примеси ведет к возможной взаимной компенсации донорных и акцепторных примесей и деградации характеристик ТД при последующей температурной обработке многопереходной структуры фотопреобразователя. Способами, позволяющими предотвратить деградацию характеристик ТД вследствие диффузии 
Таблица 1. Модельная структура: $n^{++}-\mathrm{GaAs}: \mathrm{Si} / i-\mathrm{GaAs} /$ $p^{++}$-AlGaAs : Be

\begin{tabular}{l|c|c}
\hline \multicolumn{1}{c|}{ Слои } & Концентрация, см ${ }^{-3}$ & Толщина, нм \\
\hline$n^{+}-\mathrm{GaAs}: \mathrm{Si}$ & $4 \cdot 10^{18}$ & 300 \\
$n^{++}-\mathrm{GaAs}$ & $1.7 \cdot 10^{19}$ & 10 \\
$i-\mathrm{GaAs}$ & $5 \cdot 10^{16}$ & $(0-10)$ \\
$p^{++}-\mathrm{Al}_{0.2} \mathrm{Ga}_{0.8} \mathrm{As}$ & $4 \cdot 10^{19}$ & 10 \\
$i-\mathrm{Al}_{0.2} \mathrm{Ga}_{0.8} \mathrm{As}$ & $5 \cdot 10^{16}$ & 2.5 \\
$i-\mathrm{GaAs}$ & $5 \cdot 10^{16}$ & 2.5 \\
$p^{+}-\mathrm{GaAs}: \mathrm{Be}$ & $2 \cdot 10^{19}$ & 250 \\
$p^{+}-\mathrm{GaAs}: \mathrm{Be}$ & $1 \cdot 10^{19}$ & 350000
\end{tabular}

легирующей примеси, являются снижение температуры эпитаксиального роста и включение промежуточного нелегированного $i$-слоя между вырожденными $p^{++}$и $n^{++}$-слоями.

Таким образом, полупроводниковые материалы, температурные режимы эпитаксиального роста, тип донорных и акцепторных примесей, профиль и уровни легирования, а также толщины эпитаксиальных слоев в ТД оказывают значительное влияние на электрические и оптические характеристики как самих соединительных элементов, так и многопереходных фотопреобразователей.

В данной статье представлены исследования по разработке высокоэффективных соединительных $p^{++}-i-n^{++}$-ТД на основе структур GaAs/AlGaAs, с высокой плотностью пикового туннельного тока, выращенных методом МПЭ. Указанные туннельные диоды предназначены для применения в качестве соединительных элементов в мощных многопереходных фотопреобразователях в системе GaAs/AlGaAs.

\section{2. Моделирование структуры туннельных диодов и расчет их характеристик}

Рассматривались соединительные туннельные диоды, структура которых представлена в табл. 1. Диоды различались толщиной $i$-области, которая изменялась от 0 до 10 нм. Расчеты были выполнены в рамках программного пакета Silvaco Atlas [13]. Использовалось два типа моделей: нелокального межзонного квантового туннелирования (НМКТ) и туннелирования через ловушки (ТЛ) [12-14].

Модель НМКТ описывает величину пикового туннельного тока, т.е. туннельную компоненту плотности тока, $J_{t}$, в то время как ТЛ описывает ток после ,долины“ поненты [12-17]. Для краткости приведем выражения, используемые только в модели НМКТ. Согласно данной модели, вклад в туннельный ток для электронов в диапазоне энергий $E-\Delta E / 2$ до $E+\Delta E / 2$ описывался следующим образом:

$$
\begin{aligned}
J(E)= & \frac{q k_{0} T}{2 \pi^{2} \hbar^{3}} \tau(E)\left(m_{e}^{*} \ln \left\{\frac{1+\exp \left[\left(E-E_{F l}^{e}\right) / k_{0} T\right]}{1+\exp \left[\left(E-E_{F r}^{e}\right) / k_{0} T\right]}\right\}\right. \\
& \left.-m_{h}^{*} \ln \left\{\frac{1+\exp \left[\left(E-E_{F l}^{h}\right) / k_{0} T\right]}{1+\exp \left[\left(E-E_{F r}^{h}\right) / k_{0} T\right]}\right\}\right) \Delta E,
\end{aligned}
$$

где $\tau(E)$ - вероятность квантового туннелирования, $T$ - температура, $k_{0}$ - постоянная Больцмана, $q-$ заряд электрона, $\hbar$ - приведенная постоянная Планка, $E_{F l}$ и $E_{F r}-$ уровни Ферми с левой и правой сторон от туннельного перехода, $m_{e}^{*}$ и $m_{h}^{*}$ - эффективные массы для электронов и дырок соответственно,

$$
\begin{aligned}
& m_{e}^{*}=m_{0} \sqrt{m_{e}\left(x_{\mathrm{end}}\right) m_{h}\left(x_{\mathrm{beg}}\right)}, \\
& m_{h}^{*}=m_{0} \sqrt{\left(m _ { e } \left(x_{\mathrm{beg})} m_{h}\left(x_{\mathrm{end}}\right)\right.\right.} .
\end{aligned}
$$

Вероятность квантового туннелирования рассчитывалась с использованием приближения Вентцеля-Крамерса-Бриллюэна (WKB) [18-20] в соответствии со следующим выражением:

$$
\tau(E)=\exp \left(-2 \int_{x_{\text {beg }}}^{x_{\text {end }}} k(x) d x\right),
$$

где $x_{\text {beg }}$ и $x_{\text {end }}$ - начальная и конечная точки пути туннелирования носителя заряда через барьер, соответствующие сетке пространственной дискретизации, а $k$ - модуль волнового вектора, который определяется выражениями

$$
\begin{gathered}
k(x)=\frac{k_{e} k_{h}}{\sqrt{k_{e}^{2}+k_{h}^{2}}}, \\
k_{e}(x)=\frac{1}{i \hbar} \sqrt{2 m_{0} m_{e}(x)\left[E-E_{c}(x)\right]} \\
k_{h}(x)=\frac{1}{i \hbar} \sqrt{2 m_{0} m_{h}(x)\left[E-E_{v}(x) .\right.}
\end{gathered}
$$

В выражениях (5)-(7) $k_{e}$ и $k_{h}-$ модули волновых векторов для электронов и дырок, $m_{0}-$ масса покоя электрона, $E$ - энергия носителя заряда, $E_{c}-$ энергия, соответствующая дну зоны проводимости, $E_{v}-$ энергия, соответствующая потолку валентной зоны. Согласно выражениям (5)-(7) для волнового вектора, при расчете $\tau(E)$ (4) квантовое туннелирование носителей заряда рассматривается как электронное в непосредственной близости от зоны проводимости, как дырочное в непосредственной близости от валентной зоны и как электронно-дырочное между зонами [13]. В транспортных уравнениях использовались модели безызлучательной рекомбинации Шокли-Рида-Холла (SRH), сужения ширины запрещенной зоны (BGN), а также статистика Ферми-Дирака [12-14]. 
На рис. 1 показаны результаты расчета вольт-амперной характеристики (BAX) $J(U)$ туннельного диода (кривая 1 ) при толщине $i$-слоя $0.3 \mathrm{Hм,} \mathrm{а} \mathrm{также} \mathrm{за-}$ висимости пикового значения плотности туннельного тока $J_{\text {peak }}$ от толщины $i$-слоя (кривая 2). Видно, что при отсутствии $i$-слоя максимальное значение плотности пикового туннельного тока равно $J_{\text {peak }}=90 \mathrm{~A} / \mathrm{cm}^{2}$. С ростом толщины $i$-слоя плотность пикового тока $J_{\text {peak }}$ возрастает, достигает максимума $280 \mathrm{~A} / \mathrm{cm}^{2}$ при толщине $i$-слоя $\sim 4$ нм, а затем снижается и при толщине $i$-слоя 10 нм становится меньше первоначального.

На рис. 2-4 представлены рассчитанные распределения по координате $x$ (перпендикулярно слоям) напряженности электрического поля и концентрации электро-

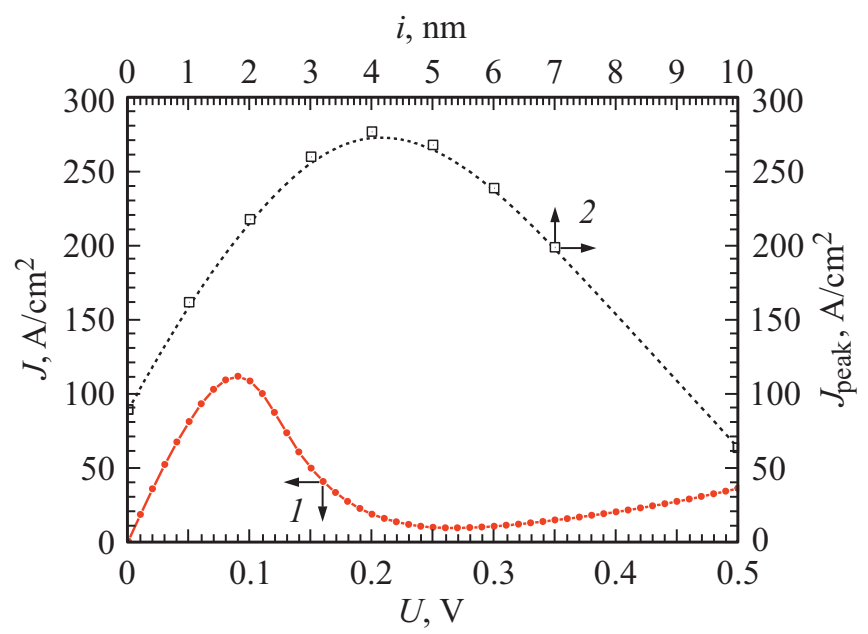

Рис. 1. Вольт-амперная характеристика соединительного $p-i-n$-ТД (табл. 1) при толщине $i$-слоя $\sim 0.3$ нм (1) и зависимость величины плотности пикового туннельного тока от толщины $i$-слоя для структуры $p-i-n$-GaAs/AlGaAs ТД (2).

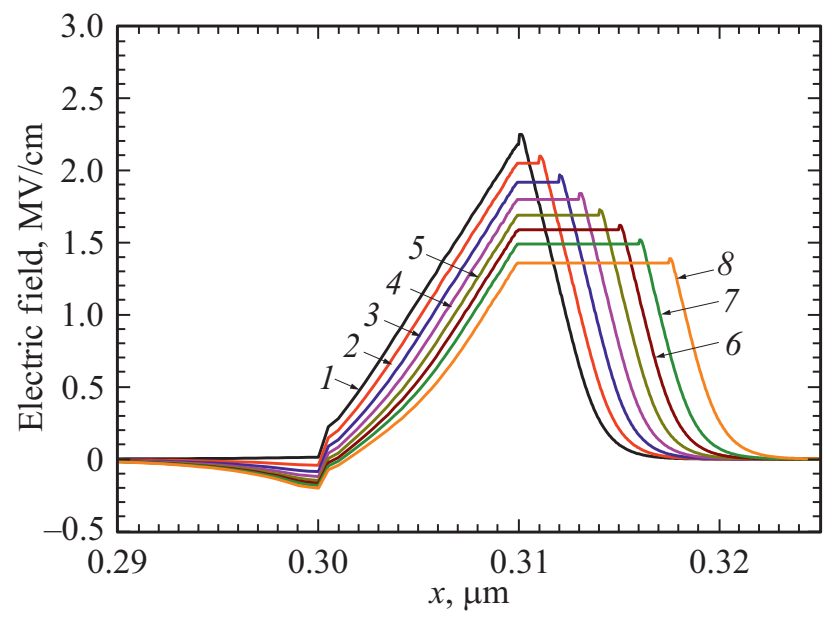

Рис. 2. Распределения напряженности электрического поля в активной области структуры $n^{+}$-GaAs $/ n^{++}-\mathrm{GaAs} /$ $i$-GaAs $/ p^{++}-\mathrm{Al}_{0.2} \mathrm{Ga}_{0.8} \mathrm{As}$ по координате $x$ перпердикулярно слоям при изменении толщины $i$-слоя от 0 до 7.5 нм. $i$, нм: $1-0,2-1,3-2,4-3,5-4,6-5,7-6,8-7.5$.

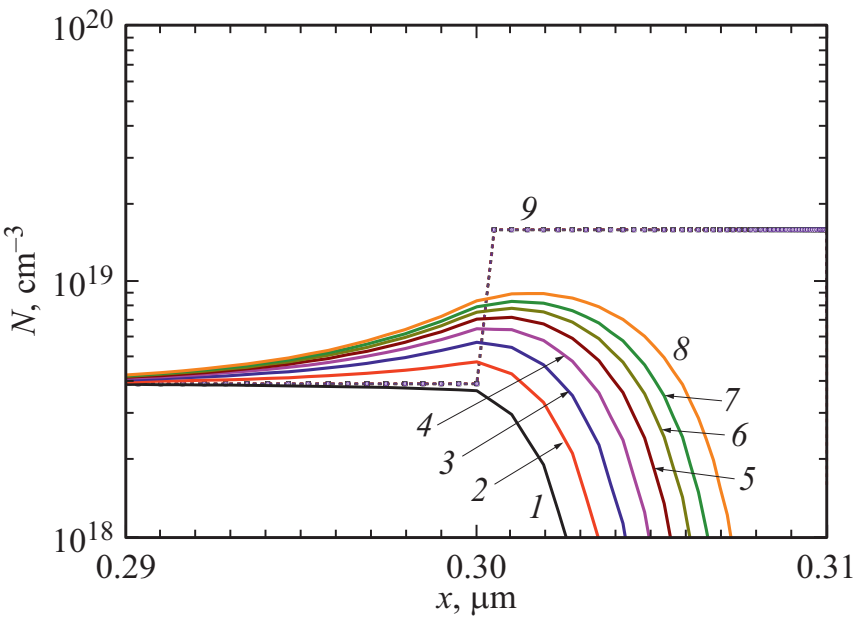

Рис. 3. Распределения концентраций свободных электронов $N$ в области $n^{+}-\mathrm{GaAs} / n^{++}$-GaAs ТД при изменении толщины $i$-слоя от 0 до 7.5 нм. $i$, нм: $1-0,2-1,3-2,4-3,5-4$, $6-5,7-6,8-7.5 ; 9-$ расчетные уровни легирования кремнием в $n^{++}$-слое $\left(N_{D}=1.7 \cdot 10^{19} \mathrm{~cm}^{-3}\right)$ и прилегающем к нему $n^{+}$-слое $\left(N_{D}=4 \cdot 10^{18} \mathrm{~cm}^{-3}\right)$.

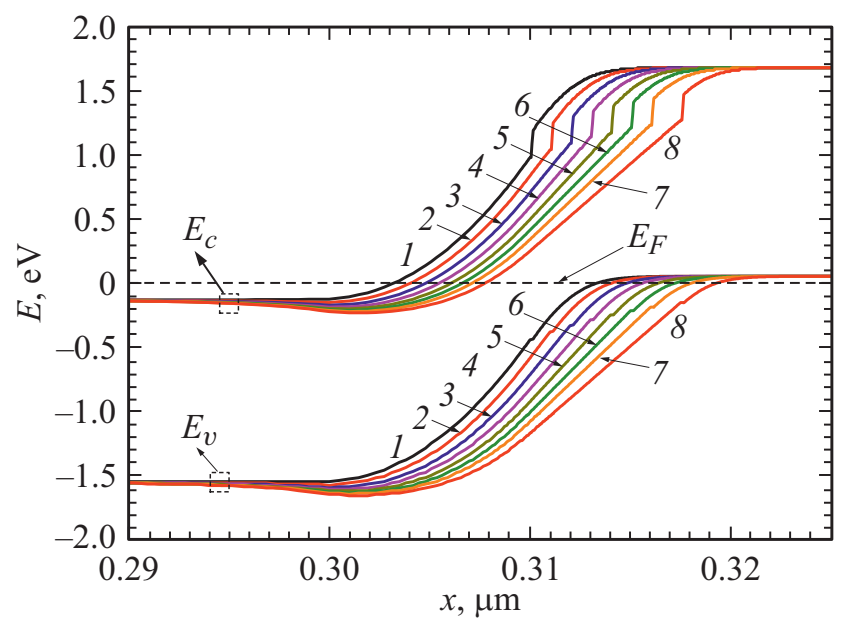

Рис. 4. Зонные диаграммы активной области $n^{+}-\mathrm{GaAs} /$ $n^{++}-\mathrm{GaAs} / i$-GaAs $/ p^{++}-\mathrm{Al}_{0.2} \mathrm{Ga}_{0.8} \mathrm{As}$ для толщины $i$-слоя от 0 до 7.5 нм при напряжении смещения $U=0$ В. $i$, нм: $1-0$, $2-1,3-2,4-3,5-4,6-5,7-6,8-7.5 . E_{F}-$ уровень Ферми.

нов в высоколегированном $n^{++}$-слое, в прилегающем к нему менее легированном изотипном слое, а также зонные диаграммы активной $p-i-n$-области исследуемой структуры при значениях толщин $i$-слоя $(i)$ от 0 до 7.5 нм. Представленные данные позволили выявить факторы, влияющие на величину туннельного тока при изменении толщины $i$-слоя.

Во-первых, при отсутствии $i$-слоя между вырожденными слоями ТД область объемного заряда, соответствующая кривой 1 на рис. 2, занимает весь вырожденный слой $n^{++}$, в результате чего концентрация электронов, могущих участвовать в процессе туннелирования, умень- 
шается от $1.7 \cdot 10^{19} \mathrm{~cm}^{-3}$ до величины концентрации примеси в прилегающем изотипном слое $4 \cdot 10^{18} \mathrm{~cm}^{-3}$ (рис. 3, кривая 1). Увеличение толщины $i$-слоя приводит к снижению максимальной величины напряженности электрического поля в области объемного заряда. Это в свою очередь приводит к уменьшению степени обеднения носителями заряда вырожденного $n^{++}$-слоя и возрастанию в нем концентрации (кривые 2-8 на рис. 3). Кроме того, увеличение концентрации электронов в $n^{++}$-области с ростом толщины $i$-слоя приводит к появлению локального минимума дна зоны проводимости (рис. 4), что приводит к увеличению диапазона энергий электронов, участвующих в туннелировании через барьер. Повышение доступных для туннелирования электронов в вырожденном $n^{++}$-слое способствует увеличению плотности туннельного тока с ростом толщины $i$-слоя.

Во-вторых, согласно зонным диаграммам активной области ТД в зависимости от толщины $i$-слоя, изображенным на рис. 4 , гетеробарьер $\mathrm{GaAs} / \mathrm{Al}_{0.2} \mathrm{Ga}_{0.8} \mathrm{As}$ обеспечивает появление скачка энергии носителей в зоне проводимости [21], что влияет на величину волнового вектора $k(x)$ и, следовательно, на величину вероятности квантового туннелирования согласно выражению (4).

В-третьих, эффективная масса электрона в тонком слаболегированном $i$-слое будет значительно ниже эффективной массы электрона в вырожденных слоях ТД [22], что также способствует увеличению пикового значения туннельного тока.

С другой стороны, увеличение толщины $i$-слоя приводит к уменьшению вероятности квантового туннелирования через барьер и, следовательно, должно вызывать снижение плотности туннельного тока. Совокупность всех этих факторов и приводит к появлению экстремума на кривой 2 на рис. 1 при толщине $i$-слоя $\sim 4$ нм.

\section{3. Эксперимент}

\section{1. Технология роста}

Экспериментальные структуры ТД выращивались технологией МПЭ в двухреакторной установке (SemiTEq, Россия). Эпитаксиальная реакторная камера для соединений $\mathrm{A}^{\mathrm{III}} \mathrm{B}^{\mathrm{V}}$ оснащена стандартными эффузивными источниками материалов $\mathrm{Ga}$ и $\mathrm{Al}$, а также $\mathrm{Si}$ и $\mathrm{Be}$ (для $n$ - и $p$-легирования соответственно). Источником мышьяка служил источник с клапаном и разложителем при температуре $560^{\circ} \mathrm{C}$ (Veeco), данная температура соответствует преобладающему потоку молекулярного мышьяка $\mathrm{As}_{4}$. Контроль ростовой поверхности осуществлялся in situ при помощи метода дифракции быстрых электронов на отражение (ДБЭО). Нагрев подложки происходил радиационным бесконтактным образом. Показатели термопары соотносились с реальными значениями температуры вблизи $510^{\circ} \mathrm{C}$ подложки через набор контрольных точек перестройки поверхности $\mathrm{GaAs}(001)$ из $(2 \times 4)$ As в $(4 \times 4)$ As при известном падающем потоке As [23].

С использованием данной установки выращены структуры туннельных диодов под номерами А и В, технологические параметры представлены в табл. 2. Эпитаксиальные структуры ТД были выращены на подложках $p$-GaAs : Be (100) с концентрацией акцепторов $N_{\mathrm{A}}=1 \cdot 10^{19} \mathrm{~cm}^{-3}$, толщиной 350 мкм и содержали буферный слой $p$-GaAs:Be с идентичным уровнем легирования. Для увеличения эффективного уровня легирования вырожденных слоев ТД применялось модулированное $\delta$-легирование кремнием и бериллием [24]. Активная область ТД представляла собой $p-i-n$-гетеропереход из слоев $p^{++}-\mathrm{Al}_{0.2} \mathrm{Ga}_{0.8} \mathrm{As}: \mathrm{Be} /$ $i-\mathrm{Al}_{0.2} \mathrm{Ga}_{0.8} \mathrm{As} / i$-GaAs $/ n^{++}-\mathrm{GaAs}: \mathrm{Si}$ с не сплошным легированием вырожденных слоев по толщине, а с периодически интегрированными $\delta$-слоями легирующей примеси Ве (с поверхностной плотностью атомов Ве $6 \cdot 10^{13} \mathrm{~cm}^{-2}$ ) или $\delta$-слоями $\mathrm{Si}$ (с поверхностной плотностью атомов $\mathrm{Si} 5 \cdot 10^{13} \mathrm{~cm}^{-2}$ ) через равные промежутки нелегированного материала $2.5 \mathrm{HM} \quad \mathrm{Al}_{0.2} \mathrm{Ga}_{0.8} \mathrm{As}$ для $p$-типа проводимости и $\mathrm{GaAs}$ для $n$-типа. Между вырожденными слоями ТД была выращена $i$-область, состоящая из двух слоев: $i$-GaAs толщиной 2.5 нм (структура А) или 5 нм (структура В) и слоя $i-\mathrm{Al}_{0.2} \mathrm{Ga}_{0.8} \mathrm{As}$ толщиной 5 нм для обеих структур (табл. 2). После активной области следовал приконтактный слой $n^{+}-\mathrm{GaAs}: \mathrm{Si}$ толщиной 300 нм со сплошным легированием и концентрацией доноров $N_{D}=4 \cdot 10^{18} \mathrm{~cm}^{-3}$.

Температура эпитаксиального роста является одним из основных параметров, влияющим на легирующие примеси $\mathrm{Si}$ и Ве в $\delta$-слоях [25]. В структурах ТД после буферного слоя $\mathrm{GaAs}: \mathrm{Si}$, формируемого при температуре $580^{\circ} \mathrm{C}$, температура подложки опускалась до $500^{\circ} \mathrm{C}$ (структура А) или $450^{\circ} \mathrm{C}$ (структура В) с целью увеличения встраиваемости легирующих примесей и подавления диффузии.

Для проверки уровней легирования вырожденных слоев в структурах ТД были проведены холловские измерения на тестовых слоях $\mathrm{Ga}(\mathrm{Al}) \mathrm{As}$ : Be и $\mathrm{GaAs}: \mathrm{Si}$ при $300 \mathrm{~K}$ со сплошным легированием, вырашенных при тех же условиях МПЭ и температурах источников легирующей примеси. Согласно проведенным измерениям, в вырожденной области, легированной $\mathrm{Si}$, с $n$-типом проводимости, уровень концентрации доноров составлял $1.7 \cdot 10^{19} \mathrm{~cm}^{-3}$, в вырожденной области, легированной $\mathrm{Be}, \mathrm{c} p$-типом проводимости, уровень концентрации акцепторов составлял $4 \cdot 10^{19} \mathrm{~cm}^{-3}$.

С помощью стандартного метода фотолитографии и химического травления на поверхности каждой выращенной структуры $p-i-n-\mathrm{GaAs} / \mathrm{AlGaAs}$ был сформирован массив диодов с диаметрами мезы 225-900 мкм и омическими контактами $\mathrm{AuGe}-\mathrm{Ni}-\mathrm{Au}, \mathrm{AgMn}-\mathrm{Ni}-\mathrm{Au}$ соответственно к $n$ - и $p$-GaAs. Температура вжигания контактов в атмосфере водорода составляла $500-520^{\circ} \mathrm{C}$.

Были проведены исследования распределения легирующих атомов примеси по толщине туннельного 
Таблица 2. Технологические параметры выращенных структур $n^{++}-\mathrm{GaAs}: \mathrm{Si} / i-(\mathrm{GaAs} / \mathrm{AlGaAs}) / p^{++}-\mathrm{AlGaAs}: \mathrm{Be}$ туннельных диодов типов А и В

\begin{tabular}{|c|c|c|c|c|c|}
\hline \multicolumn{3}{|c|}{ Структура А } & \multicolumn{3}{|c|}{ Структура В } \\
\hline Слои & $\begin{array}{c}\text { Концентрация, } \\
\mathrm{cm}^{-3}\end{array}$ & $\begin{array}{c}\text { Толщина, } \\
\text { нм }\end{array}$ & Слои & $\begin{array}{c}\text { Концентрация, } \\
\mathrm{cm}^{-3}\end{array}$ & $\begin{array}{c}\text { Толщина, } \\
\text { нм }\end{array}$ \\
\hline$n^{+}-\mathrm{GaAs}: \mathrm{Si}$ & $4 \cdot 10^{18}$ & 300 & $n^{+}-\mathrm{GaAs}: \mathrm{Si}$ & $4 \cdot 10^{18}$ & 300 \\
\hline $\begin{array}{c}n^{++}: 3 \times \delta \text {-Si } \\
\text { через } 2.5 \mathrm{HM} \\
\mathrm{GaAs}\end{array}$ & $1.7 \cdot 10^{19}$ & 10 & $\begin{array}{c}n^{++}: 3 \times \delta \text {-Si } \\
\text { через } 2.5 \mathrm{HM} \\
\mathrm{GaAs}\end{array}$ & $1.7 \cdot 10^{19}$ & 10 \\
\hline$i$-GaAs & $5 \cdot 10^{16}$ & 2.5 & $i$-GaAs & $5 \cdot 10^{16}$ & 5 \\
\hline$i-\mathrm{Al}_{0.2} \mathrm{Ga}_{0.8} \mathrm{As}$ & $5 \cdot 10^{16}$ & 5 & $i-\mathrm{Al}_{0.2} \mathrm{Ga}_{0.8} \mathrm{As}$ & $5 \cdot 10^{16}$ & 5 \\
\hline $\begin{array}{c}p^{++}: 3 \times \delta \text {-Be } \\
\text { через } 2.5 \mathrm{Hм} \\
\mathrm{Al}_{0.2} \mathrm{Ga}_{0.8} \mathrm{As}\end{array}$ & $4 \cdot 10^{19}$ & 10 & $\begin{array}{c}p^{++}: 3 \times \delta \text {-Be } \\
\text { через } 2.5 \mathrm{HM} \\
\mathrm{Al}_{0.2} \mathrm{Ga}_{0.8} \mathrm{As}\end{array}$ & $4 \cdot 10^{19}$ & 10 \\
\hline$i-\mathrm{Al}_{0.2} \mathbf{G a}_{0.8} \mathrm{As}$ & $5 \cdot 10^{16}$ & 2.5 & $i-\mathrm{Al}_{0.2} \mathbf{G a}_{0.8} \mathrm{As}$ & $5 \cdot 10^{16}$ & 2.5 \\
\hline$i$-GaAs & $5 \cdot 10^{16}$ & 2.5 & $i$-GaAs & $5 \cdot 10^{16}$ & 2.5 \\
\hline$p^{+}-\mathrm{GaAs}: \mathrm{Be}$ & $2 \cdot 10^{19}$ & 250 & $p^{+}-\mathrm{GaAs}: \mathrm{Be}$ & $2 \cdot 10^{19}$ & 250 \\
\hline$p^{+}-\mathrm{GaAs}: \mathrm{Be}$ & $1 \cdot 10^{19}$ & 350000 & $p^{+}-\mathrm{GaAs}: \mathrm{Be}$ & $1 \cdot 10^{19}$ & \\
\hline
\end{tabular}

$p-i-n$-перехода структур А и В с использованием глубинного профилирования методом динамической вторично-ионной масс-спектрометрии (ВИМС) на установке IMS-7f (САMECA, Франция). Согласно измеренным концентрационным профилям атомов, в структурах А и В наблюдается практически полная диффузия примеси Ве в $i$-слой и далее к границе $p-n$-перехода. При этом глубина проникновения атомов Ве для структуры А была выше, чем для структуры В, что связано с меньшей толщиной $i$-слоя, а также большей температурой эпитаксиального роста структуры типа А.

Таким образом, в обеих структурах чистый $i$-слой отсутствует и можно говорить лишь о некоторой его „эффективной“ толщине из-за взаимной компенсации легирующих примесей. Естественно ожидать, что „эффективная“ толщина $i$-слоя будет существенно меньше технологически заявленной толщины, указанной в табл. 2. Тем не менее, как будет показано далее, сравнение экспериментальных результатов с расчетными свидетельствует о наличии „эффективного“ $i$-слоя и его заметном влиянии на плотность пикового туннельного тока.

С целью оценки равномерности распределения толщины $i$-слоя по эпитаксиальной пластине для ТД на

Таблица 3. Экспериментальные значения емкости для туннельных диодов $n^{++}$-GaAs : $\mathrm{Si} / i$-(GaAs/AlGaAs)/ $p^{++}-\mathrm{AlGaAs}:$ Ве типов А и В

\begin{tabular}{c|c|c|c}
\hline Структура & $J_{\text {peak }}, \mathrm{A} / \mathrm{cm}^{2}$ & $\mathrm{U}, \mathrm{B}$ & $\mathrm{C}$, пФ \\
\hline $\mathrm{A}$ & 111 & 0.7 & 680 \\
& 190 & 0.7 & 314 \\
$\mathrm{~B}$ & 131 & 0.7 & 615
\end{tabular}

структурах А и В были проведены вольт-фарадные измерения. Средняя емкость $p-i-n$-диодов составила $C=600-700$ пФ (табл. 3). При этом для пластины В средняя емкость ТД оказывается заметно меньше емкости диодов на пластине А, что можно объяснить большей шириной области объемного заряда. Эти результаты согласуются с результатами описанных выше исследований концентрационных профилей методом ВИМС. С другой стороны, вольт-фарадные измерения показали двукратное снижение емкости ТД на периферии эпитаксиальной пластины структуры А, что позволяет ожидать наличия в этих диодах заметного влияния „эффективнойс6 толщины $i$-слоя.

\section{2. Исследование экспериментальных характеристик выращенных туннельных диодов}

Непосредственно на эпитаксиальных пластинах структур А и В выполнены измерения ВАХ образцов ТД с диаметром мезы 225-900 мкм, расположенных в различных местах пластины. Измерения ВАХ выполнены при положительном напряжении смещения до $0.6 \mathrm{~B}$ и комнатной температуре $(T=300 \mathrm{~K})$. На рис. 5 представлены значения пиковой плотности туннельного тока, найденные из измеренных ВАХ ТД с диаметром мезы 225 мкм для исследуемых структур А и В. Измеренные $\mathrm{BAX}$ показывают, что диапазон изменения плотности пикового туннельного тока ТД на эпитаксиальной пластине структуры А составляет от 90 до $200 \mathrm{~A} / \mathrm{cm}^{2}$, для структуры В - от 125 до $150 \mathrm{~A} / \mathrm{cm}^{2}$. В структуре А (рис. 5) наблюдается разброс по значениям плотности пикового туннельного тока на эпитаксиальной пластине 


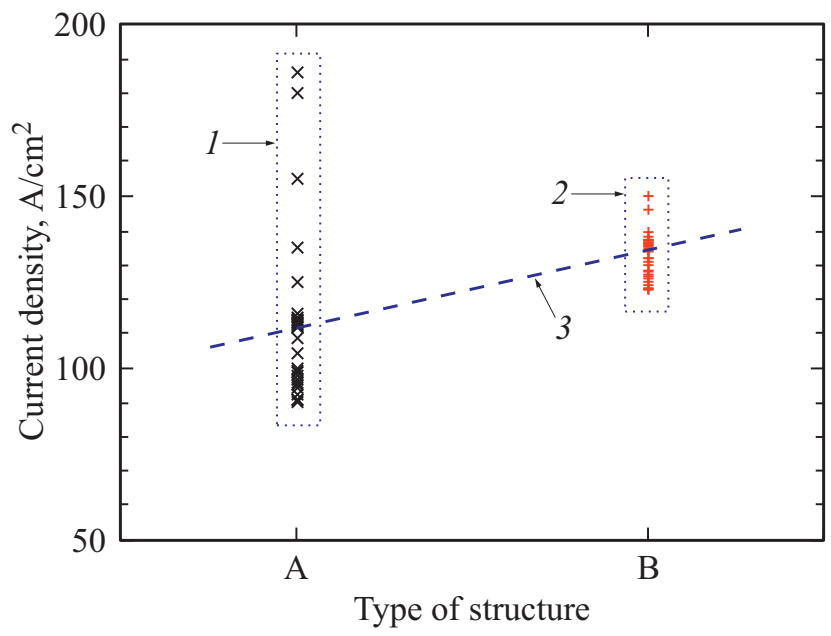

Pис. 5. Значения плотности пикового туннельного тока диодов на структурах А (1) и В (2), полученные из экспериментальных ВАХ, измеренных непосредственно на эпитаксиальной пластине. 3 - изменение усредненного значения плотности пикового туннельного тока.

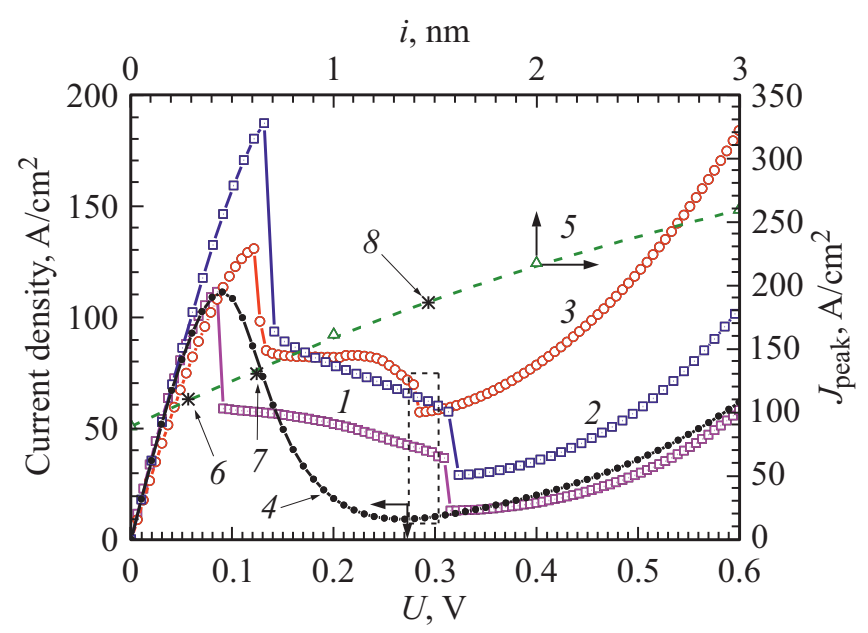

Рис. 6. Экспериментальные ВАХ ТД с диаметром мезы 225 мкм: $(1,2)$ - структура А, ВАХ со средним значением $J_{\text {peak }}$ по пластине $(1)$ и с максимальными значениями плотности пикового туннельного тока (2); 3 - структура В, ВАХ со средним значением $J_{\text {peak }}$ по пластине 4 - расчетная ВАХ ТД (табл. 1) при толщине $i$-слоя $\sim 0.3$ нм. $5-$ расчетная зависимость величин плотности пикового туннельного тока $J_{\text {peak }}$ от толщины $i$-слоя, точки 6,7 и $8-$ экспериментальные значения $J_{\text {peak }}$ для структур А и В (табл. 2).

$\Delta J_{\text {peak }} \approx 55 \%$, причем для 90\% диодов плотность пикового туннельного тока находится в более узком диапазоне, от 90 до $115 \mathrm{~A} / \mathrm{cm}^{2}$, а остальные $10 \%$ диодов, расположенные по периферии эпитаксиальной пластины, имеют существенно большую плотность пикового тока, $\sim 200 \mathrm{~A} / \mathrm{cm}^{2}$. В то же время для структуры В различие по плотности пикового туннельного тока между ТД составляет существенно меньшую величину, 20\%, и без экстремальных выбросов.
Усредненные значения плотности пикового туннельного тока, представленные на рис. 5 (кривая 3), составляют $111 \mathrm{~A} / \mathrm{cm}^{2}$ для структуры А и $131 \mathrm{~A} / \mathrm{cm}^{2}$ для структуры В (табл. 3). Значение усредненной плотности пикового туннельного тока $p-i-n$-ТД для структуры типа В с технологически закладываемой толщиной 10 нм нелегированного $i$-слоя на 15\% выше, чем плотность пикового туннельного тока ТД для структуры А с технологической закладываемой толщиной $i$-слоя 7.5 нм, что коррелирует с результатами исследований ВИМС [26] и вольт-фарадными измерениями и может быть объяснено большей „эффективной“

На рис. 6 представлены экспериментальные ВАХ для структуры А (кривая 1) и структуры В (кривая 3), а также ВАX ТД структуры А с максимальным значением плотности пикового туннельного тока (кривая 2). Видно, что экспериментальные ВАХ достаточно хорошо согласуются с ВАХ для модельной структуры (кривая 4 на этом рисунке), а среднестатистические значения плотности пикового тока хорошо ложатся на расчетную кривую зависимости $J_{\text {peak }}$ от толщины $i$-слоя (рис. 6, точки 6, 7 и 8 на кривой 5). При этом средняя эффективная толщина $i$-слоя для структуры А оказывается близкой к нулю, а для структуры В составляет $\sim 0.6$ нм. В то же время для диодов, расположенных на периферии эпитаксиальной пластины структуры А, „эффективная“ толщина $i$-слоя равна $1.5 \mathrm{HM}$, что также коррелирует с емкостными измерениями.

В наиболее общем случае ВАХ ТД при прямом напряжении смещения согласно выражению (8) включает в себя три компоненты плотности тока - туннельную, $J_{t}$, избыточную, $J_{x}$, и диффузионную, $J_{d}[15,16]$ :

$$
J_{m}=J_{t}+J_{x}+J_{d}
$$

Туннельная компонента $J_{t}$ плотности тока соответствует линейному участку ВАХ. Избыточная, $J_{x}$, и диффузионная, $J_{d}$, компоненты плотности тока соответствуют сегментам ВАХ после ,долины“. Поэтому плотность тока „долины“, а также соотношение между пиковой плотностью туннельного тока и плотностью тока долины, $J_{\text {peak }} / J_{\text {val }}$, коренным образом связаны с избыточной компонентой плотности тока ВАХ ТД. В свою очередь величина избыточной плотности тока ВАХ определяется концентрацией глубоких уровней внутри запрещенной зоны полупроводника и наличием различного рода дефектов структуры [12,15-17]. Поэтому в структуре В,

Таблица 4. Экспериментальные значения плотностей пикового тока и тока „долины“, а также их соотношения для туннельных диодов $n^{++}$-GaAs : $\mathrm{Si} / i-(\mathrm{GaAs} / \mathrm{AlGaAs}) / p^{++}-\mathrm{AlGaAs}: \mathrm{Be}$ типов А и В

\begin{tabular}{c|c|c|c}
\hline Структура & $J_{\text {peak }}, \mathrm{A} / \mathrm{cm}^{2}$ & $J_{\text {val }}, \mathrm{A} / \mathrm{cm}^{2}$ & $J_{\text {peak }} / J_{\text {val }}$, отн. ед \\
\hline $\mathrm{A}$ & 111 & 13 & 8.5 \\
$\mathrm{~B}$ & 131 & 57 & 2.3
\end{tabular}


выращенной при меньшей температуре, на BAX (рис. 6, кривая 3) наблюдаются бо́льшие значения усредненной плотности тока долины $J_{\text {val }}$ и меньшие соотношения $J_{\text {peak }} / J_{\text {val }}$ по сравнению со структурой А, выращенной при большей температуре, $500^{\circ} \mathrm{C}$, (табл. 4) [27].

\section{4. Заключение}

Выполнено численное и экспериментальное исследование характеристик соединительных туннельных $p-i-n$-диодов на основе гетероструктуры $n^{++}$-GaAs/ $i$-GaAs $/ p^{++}-\mathrm{Al}_{0.2} \mathrm{Ga}_{0.8} \mathrm{As}$.

С использованием численного моделирования установлено, что зависимость плотности пикового тока туннельного $p-i-n$-диода от толщины $i$-слоя является немонотонной. Плотность пикового тока сначала возрастает, достигает максимума, а затем снижается из-за увеличения толщины потенциального барьера, через который туннелируют носители заряда. Выявлены факторы, влияющие на величину туннельного тока при изменении толщины $i$-слоя. Для рассмотренных модельных $p-i-n$-структур определена оптимальная толщина $i$-слоя, при которой плотность пикового тока достигает $280 \mathrm{~A} / \mathrm{cm}^{2}$.

Выращены туннельные $p-i-n$-диоды со значениями плотности пикового тока до $200 \mathrm{~A} / \mathrm{cm}^{2}$. Получено достаточно хорошее согласие между расчетными и экспериментальными ВАХ туннельных диодов.

\section{Благодарности}

Выражаем благодарность П.Б. Родину, М.И. Векслеру и Н.И. Подольской за консультации и полезные советы при расчетах и использовании программы Silvaco Atlas. Исследования ВИМС проводились с использованием оборудования ЦКП „Материаловедение и диагностика в передовых технологиях“ (ФТИ им. А.Ф. Иоффе).

\section{Конфликт интересов}

Авторы заявляют, что у них нет конфликта интересов.

\section{Список литературы}

[1] W. Guter, J. Schöne, S.P. Philipps, M. Steiner, G. Sefer, A. Wekkeli, E. Welser, E. Oliva, A.W. Bett, F. Dimroth. Appl. Phys. Lett., 94, 223 (2009).

[2] https://www.ise.fraunhofer.de/en/press-media/press-releases/2014/ new-world-record-for-solar-cell-efficiency-at-46-percent.html

[3] E.C. Warmann, C. Flowers, J. Lloyd, C.N. Eisler, M.D. Escarra, H.A. Atwater. Energy Sci. Engin., 5, 69 (2017).

[4] V.S. Kalinovskii, E.V. Kontrosh, G.V. Klimko, T.S. Tabarov, S.V. Ivanov, V.M. Andreev. Techn. Phys. Lett., 44, 1013 (2018).

[5] US patent No.: US 2018/03115879 A1, November 1, 2018.

[6] W. Walukiewicz. Phys. B: Condens. Matter, 302-303, 123 (2001).
[7] W. Walukiewicz. Mater. Sci. Engin. B, 66, 39 (1999).

[8] H. Satoh, T. Imai. Jpn. J. Appl. Phys., 7 (8), 875 (1968).

[9] E.F. Schubert, J.M. Kuo, R.F. Kopf, H.S. Luftman, L.C. Hopkins, N.J. Sauer. J. Appl. Phys., 67 (4), 1969 (1990).

[10] D.L. Miller, P.M. Asbeck. J. Appl. Phys., 57, 1816 (1985).

[11] P. Enquist, G.W. Wicks, L.F. Eastman, C. Hitzman. J. Appl. Phys., 58, 4130, (1985).

[12] M. Baudrit, C. Algora. IEEE Trans. Electron Dev., 57 (11), 2564 (2010).

[13] ATLAS User's Manual (Silvaco, Santa Clara, CA, Nov. 2015).

[14] M. Hermle, G. Lertay, S.P. Philipps, A.W. Bett. Prog. Photovolt.: Res. Appl., 16, 409 (2008).

[15] L. Esaki. IEEE Trans. Electron Dev., Ed-23 (7), 644 (1976).

[16] M. Oehme, M. Šarlija, D. Hähnel, M. Kaschel, J. Werner, E. Kasper. IEEE Trans. Electron Dev., 57 (11), 2857 (2010).

[17] T.P. Brody. J. Appl. Phys., 33 (1), 100 (1962).

[18] G. Wentzel. Zeits. f. Phys., 38, 518 (1926).

[19] H.A. Kramers. Zeits. f. Phys., 39, 828 (1926).

[20] L. Brillouin. Comptes Rendus, 183 (1), 24 (1926).

[21] И.И. Абрамов, И.А. Гончаренко, Н.В. Коломейцева. ФТП, 41 (11), 1391 (2007).

[22] В.А. Богданова, Н.А. Давлеткильдеев, Н.А. Семиколенова, Е.Н. Сидоров. ФТП, 36 (4), 407 (2002).

[23] В.В. Преображенский, М.А. Путято, Б.Р. Семягин. ФТП, 36 (8), 897 (2002).

[24] K. Kohler, P. Ganser, M. Maier. J. Cryst. Growth, 127, 720 (1993).

[25] E.F. Schubert. J. Vac. Sci. \& Technol. A, 8, 2980 (1990). doi: 10.1116/1.576617.

[26] П.Н. Брунков, А.А. Гуткин, М.Э. Рудинский, О.И. Ронжин, А.А. Ситникова, А.А. Шахмин, Б.Я. Бер, Д.Ю. Казанцев, А.Ю. Егоров, В.Е. Земляков, С.Г. Конников. ФТП, 45 (6), 829 (2011).

[27] А.А. Пастор, У.В. Прохорова, П.Ю. Сердобинцев, В.В. Чалдышев, М.А. Яговкина. ФТП, 47 (8), 2 (2013).

Редактор Л.В. Шаронова

\section{Development and investigation of tunnel $p-i-n$ GaAs/AIGaAs diodes for multi-junction converters of high-power laser radiation}

\author{
V.S. Kalinovskii, E.V. Kontrosh, G.V. Klimko, S.V. Ivanov, \\ V.S. Yuferev, B.Y. Ber, D.Y. Kazantsev, V.M. Andreev \\ loffe Institute, \\ 194021 St. Petersburg, Russia
}

\begin{abstract}
Fabrication of connecting tunnel diodes with high peak tunnel current density exceeding the short-circuit current density of photoactive $p-n$ junctions is an important task in development of multi-junction III-V photovoltaic converters of high-power optical radiation. Based on the results of a numerical simulation of tunnel diode current-voltage characteristics, a method is suggested for raising the peak tunnel current density by connecting a thin undoped $i$-type layer with thickness of several nanometers between the degenerate layers of a tunnel diode. The method of molecular-beam epitaxy was used to grow $p-i-n$ $\mathrm{GaAs} / \mathrm{Al}_{0.2} \mathrm{Ga}_{0.8} \mathrm{As}$ structures of connecting tunnel diodes with peak tunnel current density of up to $200 \mathrm{~A} / \mathrm{cm}^{2}$.
\end{abstract}

